\title{
PERFORMANCE ANALYSIS OF DYNAMIC LIGHTPATH CONFIGURATION FOR SYMMETRIC WDM RING NETWORKS
}

\author{
Takuji Tachibana Shoji Kasahara \\ Nara Institute of Science and Technology
}

(Received September 29, 2003; Revised April 22, 2004)

\begin{abstract}
In this paper, we consider a dynamic lightpath configuration method where a lightpath supports multiple label switched paths (LSPs). In this method, lightpaths are established according to the congestion state of node and are released after some holding time. For the performance evaluation of the method for symmetric WDM ring networks, we consider analytical models in light and heavy traffic cases. In the light traffic case, a single node in the WDM ring network is modeled as a multiple queueing system, while $\mathrm{M} / \mathrm{G} / 1 / \mathrm{K}$ and $\mathrm{M} / \mathrm{G} / \mathrm{c} / \mathrm{c}$ queues are used for the heavy traffic case. In both cases, loss probability of packet flow and wavelength utilization factor are derived. Numerical examples show that our analytical models in both cases are useful to predict the loss probability of packet flow and the wavelength utilization factor.
\end{abstract}

Keywords: Queue, WDM networks, dynamic lightpath configuration, MPLS

\section{Introduction}

Recent rapid growth of the Internet requires evermore bandwidth due to multimedia applications such as Voice over IP (VoIP), video conference, and video-on-demand. Wavelength division multiplexing (WDM) networks can support hundreds Gbps on a single optical fiber by multiplexing wavelengths into the fiber. In the future, the number of wavelengths in a fiber will be increased to a thousand wavelengths and several Tbps transmission will be realized. Therefore, the WDM networks are attractive for the infrastructure of the next generation Internet $[2-4,6,7,9,12,14,15]$.

Optical add/drop multiplexer (OADM) selectively adds and drops a wavelength to establish an all-optical connection in WDM ring networks shown in Figure 1. The all-optical connection is called lightpath and it is established between any pair of OADMs as shown in Figure 2. The wavelengths to be added and dropped are pre-selected in each OADM $[5,10,13]$ and the lightpath configuration is not changed frequently. However, when traffic pattern changes over short time-scales, the static lightpath configuration degrades the performance of the network [18]. If the wavelengths are dynamically added and dropped, the high utilization of wavelengths and small packet loss probability are expected [1].

In [20], a lightpath configuration method for the OADM has been proposed. In the method, a lightpath is dynamically established according to the congestion state of a node, however, the release of the established lightpath is not taken into consideration. In [16], we have considered a dynamic lightpath configuration method with which a lightpath is established according to the congestion state of the node and is released when there are no packets to be transmitted with the lightpath. Note that both lightpath establishment and release times are overhead and the lightpath can not be utilized during those times. Therefore, frequent establishment or release of lightpath may decrease the utilization of 


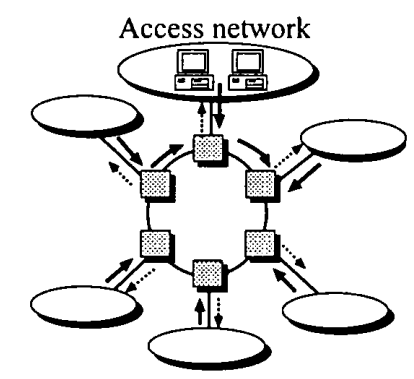

Figure 1: Ring network model

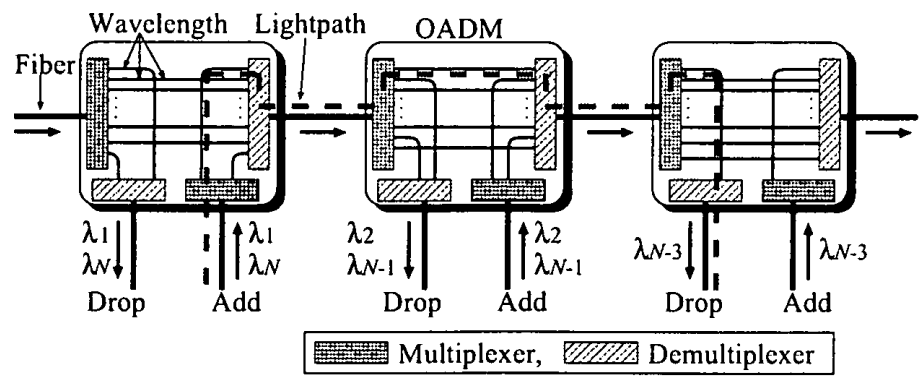

Figure 2: Optical add/drop multiplexer

wavelengths. In [16], we have introduced a design parameter called extra holding time and when there are no packets to be transmitted with some lightpath, the lightpath is still held during the extra holding time. The lightpath is released if there are no arriving packets during the extra holding time. Numerical examples in [16] have shown that the dynamic lightpath configuration provides smaller loss probability than the static lightpath configuration in asymmetric ring networks.

In [16], a lightpath is established for the transmission of a packet. In general, because the cost of the lightpath establishment and release is high, a lightpath is used for packet flows which consist of consecutive packets with the same destination network address or the same-destination label-switching router (LSR) in some access network connected to the other WDM node. In this paper, we consider the case where a lightpath supports multiple label switched paths (LSPs) and the LSP is used to transmit the packet flow from an access network to the other access network (see Figure 1).

In order to evaluate the performance of the dynamic lightpath configuration for packetflow transmission, we consider a symmetric WDM ring network under two traffic conditions: light and heavy ones. In the light traffic case, we model the dynamic lightpath configuration system as a continuous-time Markov chain to take into account the lightpath establishment/release time. In the heavy traffic case, established lightpaths are likely to be held for a while and those are rarely established or released. Therefore, we consider an $M / G / 1 / K$ and multiple $M / G / c / c$ queues for modeling the system in the heavy traffic case. In both cases, a packet flow which consists of consecutive packets is considered as a customer, and derive the loss probability of packet flow and wavelength utilization factor.

Note that in the dynamic lightpath configuration considered in the paper, the lightpath establishment and release do not occur frequently in the symmetric ring network. Therefore, our performance analysis for the symmetric ring network provides the worst case analysis and is useful for the asymmetric ring network.

The rest of the paper is organized as follows. Section 2 describes the lightpath configuration method in detail, and in Section 3, the ring network model is presented. The performance analysis of the method in the light traffic case is shown in Section 4 and the performance analysis in the heavy traffic case is presented in Section 5. Numerical examples are given in Section 6 and finally, conclusions are presented in Section 7.

\section{Dynamic Lightpath Configuration Method}

In this section, we summarize the dynamic lightpath configuration method where multiple LSPs are supported in a lightpath $[16,20]$. Each node in a WDM network consists of an OADM and an LSR as shown in Figure 3, and the nodes are connected with optical fibers 


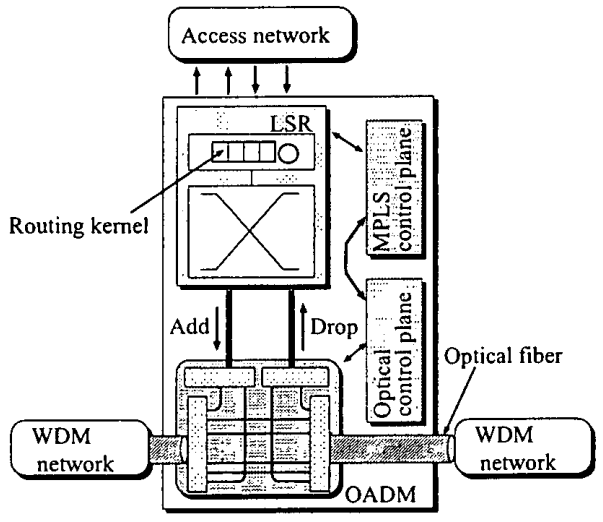

Figure 3: Node architecture

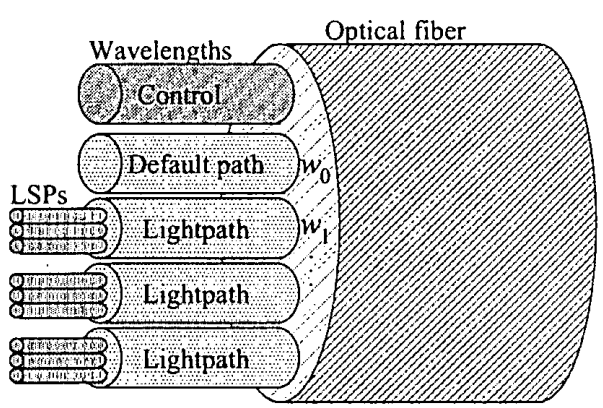

Figure 4: Data transmission structure

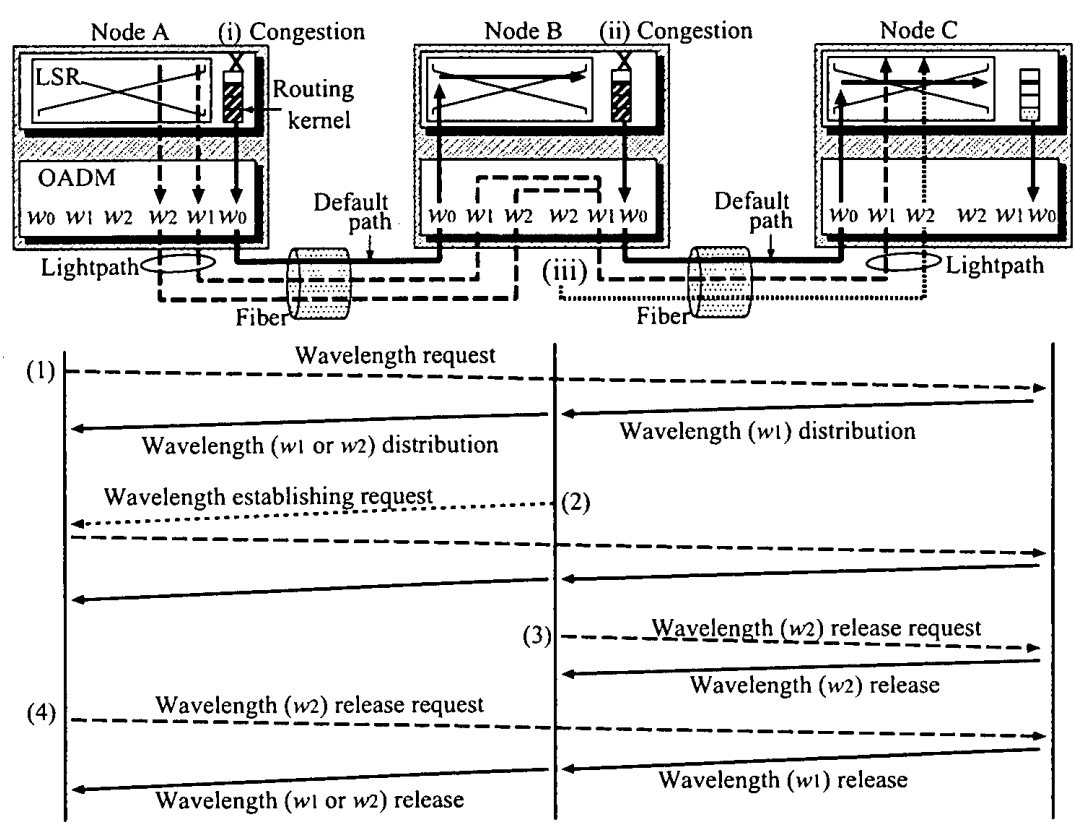

Figure 5: Dynamic lightpath configuration

as shown in Figure 4. The procedure of lightpath configuration is as follows (see Figure 5).

For simplicity, we consider a tandem network with three nodes, namely, the nodes A, B, and C. Each node is connected to its own access network through the LSR. Suppose $W+1$ wavelengths are multiplexed into an optical fiber in the WDM network. Among $W+1$ wavelengths, the $W$ wavelengths are used to transmit data traffic and one is dedicated to distribute control traffic (see Figure 4). Let $w_{i}(i=0, \cdots, W-1)$ denote the $i$ th wavelength for data traffic.

The wavelength $w_{0}$ is used for the transmission to adjacent nodes (from $\mathrm{A}$ to $\mathrm{B}$ and from $\mathrm{B}$ to $\mathrm{C}$ in Figure 5). We call $w_{0}$ the default path in the following. The default path only supports hop by hop transmission. Packets transmitted with the default path arrive at the layer 3 routing kernel in the LSR. At the routing kernels in the source and intermediate nodes, packets are routed to the next node. At the routing kernel in the destination node, on the other hand, packets are routed to the access network. 


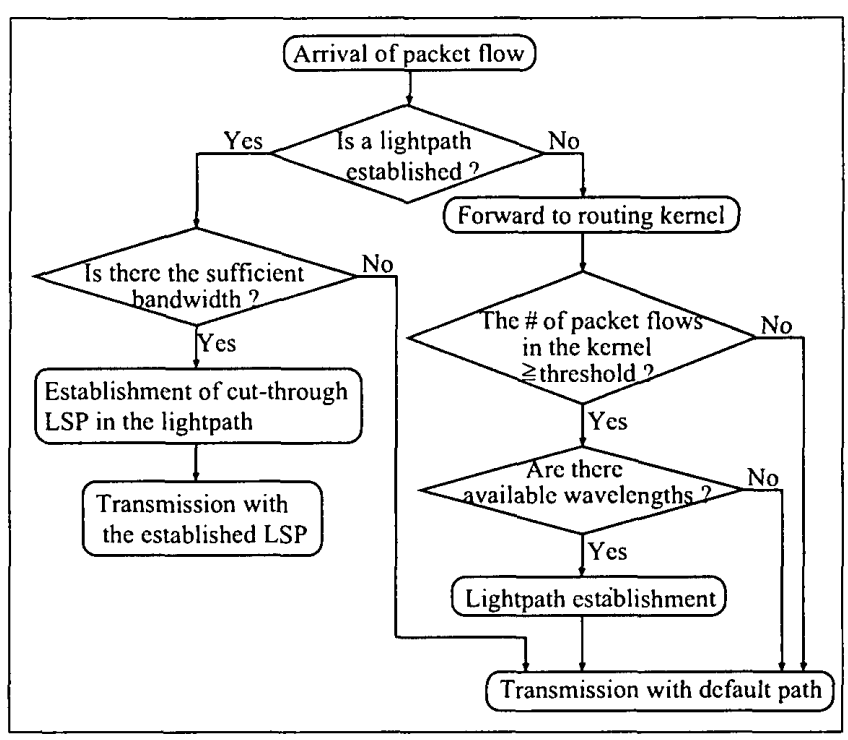

Figure 6: Flowchart of lightpath establishment

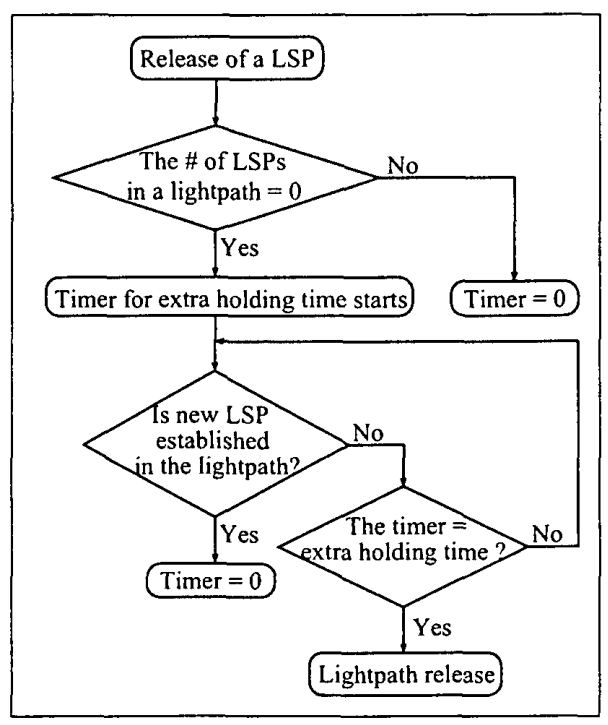

Figure 7: Flowchart of lightpath release

Other $W-1$ wavelengths are used for lightpaths which connect any pair of source and destination nodes. Those lightpaths are dynamically established and released between the source and destination nodes according to congestion states in the source and intermediate nodes along the path. An established lightpath contains multiple cut-through LSPs which have the same source and destination OADMs but have the different pairs of source and destination network addresses or the different pairs of LSRs in the access networks connected to those nodes.

When the first packet in the packet flow whose destination is in the node C's access network arrives at the node A from the A's access network, the LSR of the node A selects a wavelength with which the packet flow is transmitted. If there exists an established lightpath between the two nodes, a new cut-through LSP is established in the lightpath. If the establishment of the cut-through LSP fails due to the shortage of available bandwidth in the lightpath, the packet flow is forwarded to the routing kernel and transmitted to the destination through the default path [11].

In the dynamic lightpath configuration method, a buffer in the routing kernel of the LSR has a pre-specified threshold [20]. If the amount of packets in the buffer becomes equal to or greater than the threshold, the LSR regards the routing kernel as being in congestion and decides to establish a new lightpath between the source and destination nodes for the packet flow. This happens when the packet flow transmitted from the nodes $\mathrm{A}$ to $\mathrm{C}$ triggers congestion at the node $\mathrm{A}$, or when it triggers congestion at the node $\mathrm{B}$.

In both cases, a lightpath is established as shown in Figure 5 (1) and (2) [20]. Each node has the information of current lightpath configuration and the new lightpath configuration is performed based on the information. If the same wavelength can not be available at consecutive links, the wavelength conversion may be required at the corresponding node [13]. If no wavelengths are available at the node, the lightpath configuration fails.

When no IP packets are transmitted with the LSP during a given time interval, the node releases the LSP. When the lightpath established between the nodes $B(A)$ and $C$ (C) becomes idle, the timer for the extra holding time in the source node B (A) starts. 
The lightpath is released if the extra holding time is over and no LSP is established in the lightpath (Figure 5 (3) (Figure $5(4))$ ).

The procedures of the lightpath establishment and release are shown in Figures 6 and 7 , respectively.

\section{Network Model}

For the performance analysis of the dynamic lightpath configuration, we consider a symmetric WDM ring network with $L$ nodes, shown in Figure 1. Each node consists of an OADM and an LSR, and lightpaths are established or released according to the dynamic lightpath configuration method. In addition, each node is connected to its own access network through the LSR. For simplicity, we assume that the unit of transmission is a packet flow and that multiple lightpaths between any pair of nodes are not permitted. Moreover, we assume that the LSP is released after all IP packets in a packet flow are transmitted.

The number of wavelengths available at each node is $W$ and all wavelengths can be converted regardless of any wavelength pairs. One of the $W$ wavelengths is for the default path and the others are for the lightpaths which are dynamically established and released. The $W-1$ wavelengths are numbered from 1 to $W-1$ and a lightpath is established with one of the $W-1$ wavelengths according to the first-fit strategy in which the available wavelength with the smallest index number is selected.

Moreover we assume that the size of a packet flow is exponentially distributed with the mean $\delta$ bits and that the destination of each packet flow is equally likely. This implies that the destination of packet flow which arrives at node $i$ is node $j(j \neq i)$ with probability $1 /(L-1)$. Packet flows sent to some destination arrive at a node according to a Poisson process with rate $\lambda$. Since there are $L-1$ destinations for each node, packet flows arrive at the node from its access network according to a Poisson process with rate $(L-1) \lambda$. In this ring network, packet flows are transmitted in clockwise direction. Since the network is symmetric, we focus on a node in the network and consider the performance of the dynamic lightpath configuration method.

We assume that $W$ wavelengths have the same bandwidth $B$ bps, i.e., the bandwidth of an established lightpath also has $B$ bps. In addition, all established cut-through LSPs have the same bandwidth equal to $B_{l}$ bps. Therefore, a lightpath supports up to $K_{l}=\left\lfloor B / B_{l}\right\rfloor$ cut-through LSPs where $\lfloor x\rfloor$ is the maximum integer smaller than or equal to $x$.

Let $K_{r} \delta$ [bits] denote the capacity of the layer 3 routing kernel in the LSR. Here, the capacity consists of a waiting room in which packet flows are stored for transmission, and a server where a packet flow is in transmission. Let $T_{h} \delta$ [bits] denote the pre-specified value of the threshold for the routing kernel. For simplicity of the analysis, we assume that the unit of both $K_{r}$ and $T_{h}$ is the number of packet flows.

\section{Performance Analysis in the Light Traffic Case}

In this and the following sections, we analyze the performance of the dynamic configuration for a symmetric WDM ring network. This section is devoted to the analysis in the light traffic case and the next section to that in the heavy traffic case.

\subsection{System model}

In the light traffic case, the establishment and release of lightpaths may greatly affect the performance of the dynamic lightpath configuration method. Thus we consider a multiple queueing system under light traffic as shown in Figure 8. In this network model, there are $W$ queues in a node: one is for layer 3 routing kernel and the other $W-1$ queues are 


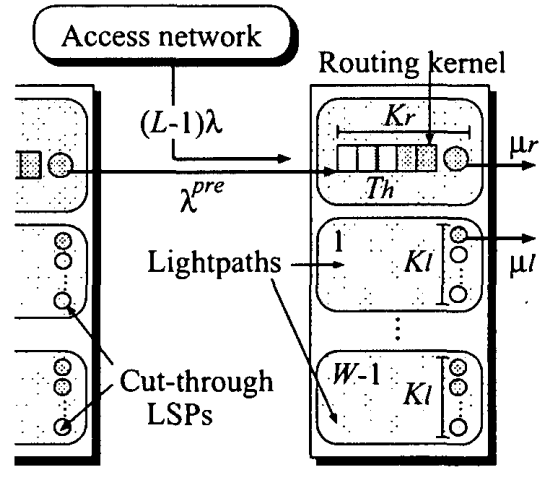

Figure 8: Ring node model with light traffic

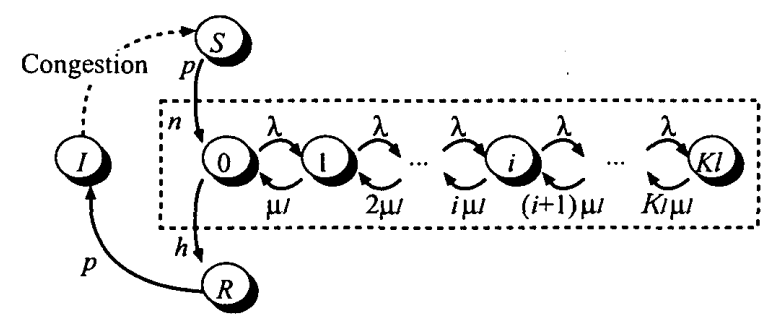

Figure 9: State transition diagram for a lightpath

for the lightpaths which are dynamically used according to the congestion of the routing kernel. Here a lightpath supports $K_{l}$ cut-through LSPs. In the light traffic case, we assume that the transmission times of packet flows for the routing kernel and cut-through LSP are exponentially distributed with rates $\mu_{r}$ and $\mu_{l}$, respectively. Because the processing speed of the routing kernel is $B_{r}$ bps and the size of a packet flow is $\delta$ bits, the mean transmission time of the routing kernel is given by $1 / \mu_{r}=\delta / B_{r}$, and that of cut-through LSP is given by $1 / \mu_{l}=\delta / B_{l}$. Note that $B_{r} \leq B$ and $B_{l} \leq B$ where $B$ is the bandwidth of a lightpath. We also assume that the lightpath establishment/release time and the extra holding time are exponentially distributed with rates $p$ and $h$, respectively.

We have two kinds of packet flows that arrives at the node: one is from the access network and the other is from the previous node. As shown in the above, we assume that packet flows arrive at the node from the access network according to a Poisson process with rate $(L-1) \lambda$.

Next we consider packet flow traffic from the previous node. Since the packet flow arrives at the routing kernel depending on the congestion state and the queue size of the routing kernel is finite, our ring network is not an open Jackson queueing network. However, due to light traffic, a newly arriving packet flow is hardly lost and is likely to be transmitted through the default path. Therefore we can approximate the arrival process from the previous node with the similar approach to the analysis of open Jackson network $[8,19]$.

The packet flow transmitted with the default path from the previous node arrives at the routing kernel in the tagged node, and then it is routed to the node's access network or the next node. Let $\lambda^{\text {pre }}$ denote the arrival rate at the routing kernel in the tagged node. Considering the transmissions originated from the other $(L-1)$ nodes, we obtain

$$
\lambda^{\text {pre }}=\frac{L(L-1)}{2} \lambda
$$

We assume that the packet flow arrival process from the previous node to the routing kernel is Poisson with rate $\lambda^{\text {pre }}$. Thus the whole arrival rate of packet flows at the node, $\lambda_{\text {all }}$, is given by

$$
\lambda_{\text {all }}=(L-1) \lambda+\lambda^{\text {pre }}=\frac{(L+2)(L-1)}{2} \lambda .
$$




\subsection{Performance analysis}

Let $l_{i}(1 \leq i \leq W-1)$ denote the $i$ th lightpath dynamically established and released at the node. We define the state of $l_{i}$ at $t$ as follows.

$$
J_{l_{i}}(t)= \begin{cases}n,\left(n=0, \cdots, K_{l}\right), & \text { if } l_{i} \text { is busy and } n \text { cut-through LSPs are established, } \\ I, & \text { if } l_{i} \text { is idle, } \\ S, & \text { if } l_{i} \text { is being established, } \\ R, & \text { if } l_{i} \text { is being released. }\end{cases}
$$

Let $N_{r}(t)$ denote the number of packet flows in the routing kernel at $t$. Then we define the state of the system at $t$ as $\left(N_{r}(t), J_{l}(t)\right)$, where

$$
J_{l}(t)=\left(J_{l_{1}}(t), \cdots, J_{l_{W-1}}(t)\right)
$$

The state transition diagram for $l_{i}$ is illustrated in Figure 9 . Note that in this figure, $1 / p$ and $1 / h$ denote the mean lightpath establishment/release time and the mean extra holding time, respectively. Let $U$ denote the whole state space of $\left(N_{r}(t), J_{l}(t)\right)$ and $U_{l}$ the space comprised of $\boldsymbol{J}_{l}(t)$.

We define $M_{l}^{B}\left(J_{l}(t)\right)$ as the number of busy lightpaths in the state $\left(N_{r}(t), J_{l}(t)\right)$. $M_{l}^{B}\left(\boldsymbol{J}_{l}(t)\right)$ is given by

$$
M_{l}^{B}\left(J_{l}(t)\right)=\sum_{i=1}^{W-1} \sum_{n=0}^{K_{l}} 1_{\left\{J_{l_{i}}(t)=n\right\}}
$$

where $1_{\{X\}}$ is the indicator function of event $X$. Similarly, we define $M_{l}^{K_{l}}\left(J_{l}(t)\right)$ as the number of lightpaths where $K_{l}$ cut-through LSPs are established. Let $M_{l}^{I}\left(\boldsymbol{J}_{l}(t)\right)$ denote the number of idle lightpaths. We have

$$
\begin{aligned}
M_{l}^{K_{l}}\left(J_{l}(t)\right) & =\sum_{i=1}^{W-1} 1_{\left\{J_{l_{i}}(t)=K_{l}\right\}}, \\
M_{l}^{I}\left(J_{l}(t)\right) & =\sum_{i=1}^{W-1} 1_{\left\{J_{l_{i}}(t)=I\right\}} .
\end{aligned}
$$

In the remainder of this section, the argument $t$ is omitted since we consider the system in equilibrium.

The transition rate from the state $\left(N_{r}, \boldsymbol{J}_{l}\right)$ is shown in Table 1 . Here, $\boldsymbol{e}_{i}$ is a $1 \times(W-1)$ vector whose $i$ th element is one and the others are zero. $i_{I}^{\min }$ in Table 1 is defined as

$$
i_{I}^{\min }=\min \left\{i ; J_{l_{i}}=I, 1 \leq i \leq W-1\right\} .
$$

and $\left(\boldsymbol{J}_{l}(t)\right)$ is omitted from $M_{l}^{x}\left(\boldsymbol{J}_{l}(t)\right),\left(x=B, K_{l}, I\right)$.

Finally, let $\pi\left(N_{r}, \boldsymbol{J}_{l}\right)$ denote the steady state probability of $\left(N_{r}, \boldsymbol{J}_{l}\right) . \pi\left(N_{r}, \boldsymbol{J}_{l}\right)$ is uniquely determined by equilibrium state equations and the following normalized condition

$$
\sum_{\left(N_{r}, \boldsymbol{J}_{l}\right) \in U} \pi\left(N_{r}, \boldsymbol{J}_{l}\right)=1
$$

In Appendix $\mathrm{A}$, we present the equilibrium state equations in the case of $W=2$.

With $\pi\left(K_{r}, J_{l}\right)$, the packet-fiow loss probability $P_{\text {loss }}$ is yielded as

$$
P_{\text {loss }}=\sum_{\left(K_{\mathrm{r}}, \boldsymbol{J}_{l}\right) \in U}\left\{1-M_{l}^{B}\left(\boldsymbol{J}_{l}\right) \frac{\lambda}{\lambda_{\text {all }}}+M_{l}^{K_{l}}\left(\boldsymbol{J}_{l}\right) \frac{\lambda}{\lambda_{\text {all }}}\right\} \pi\left(K_{r}, \boldsymbol{J}_{l}\right) .
$$


Table 1: State transition rate in ring network model

\begin{tabular}{cccc}
\hline $\begin{array}{c}\text { Number of } \\
\text { idle lightpaths }\end{array}$ & $\begin{array}{c}\text { Current state } \\
\left(N_{r}, \boldsymbol{J}_{l}\right)\end{array}$ & Next state & Transition rate \\
\hline \hline$M_{l}^{I}>0$ & $N_{r}<T_{h}$ & $\left(N_{r}+1, \boldsymbol{J}_{l}\right)$ & $\lambda_{\text {all }}-\left(M_{l}^{\left.\bar{B}-M_{l}^{K_{l}}\right) \lambda}\right.$ \\
& $T_{h} \leq N_{r}<K_{r}$ & $\left(N_{r}+1, \boldsymbol{J}_{l}\right), J_{l_{\text {min }}}=S$ & $\lambda_{\text {all }}-M_{l}^{B} \lambda$ \\
& $T_{h} \leq N_{r}<K_{r}$ & $\left(N_{r}+1, \boldsymbol{J}_{l}\right)$ & $M_{l}^{K_{l} \lambda}$ \\
& $N_{r}=K_{r}$ & $\left(N_{r}, \boldsymbol{J}_{l}\right), J_{l_{\text {min }}}=S$ & $\lambda_{\text {all }}-M_{l}^{B} \lambda$ \\
& $N_{r}>0$ & $\left(N_{r}-1, \boldsymbol{J}_{l}\right)$ & $\mu_{r}$ \\
\hline$M_{l}^{I}=0$ & $N_{r}<K_{r}$ & $\left(N_{r}+1, \boldsymbol{J}_{l}\right)$ & $\lambda_{\text {all }}-\left(M_{l}^{B}-M_{l}^{K_{l}}\right) \lambda$ \\
& $N_{r}>0$ & $\left(N_{r}-1, \boldsymbol{J}_{l}\right)$ & $\mu_{r}$ \\
\hline \hline State of & Current state & & \\
lightpaths & $\left(N_{r}, \boldsymbol{J}_{l}\right)$ & Next state & Transition rate \\
\hline \hline$J_{l_{i}}=S$ & $\left(N_{r}, \boldsymbol{J}_{l}\right)$ & $\left(N_{r}, \boldsymbol{J}_{l}\right), J_{l_{i}}=0$ & $p$ \\
\hline$J_{l_{i}}=n$ & $n<K_{l}$ & $\left(N_{r}, \boldsymbol{J}_{l}+\boldsymbol{e}_{i}\right)$ & $\lambda$ \\
& $n>0$ & $\left(N_{r}, \boldsymbol{J}_{l}-\boldsymbol{e}_{i}\right)$ & $n \mu_{l}$ \\
\hline$J_{l_{i}}=R$ & $n=0$ & $\left(N_{r}, \boldsymbol{J}_{l}\right), J_{l_{i}}=R$ & $h$ \\
\hline
\end{tabular}

We define $P_{\text {light }}$ as the lightpath utilization factor and $P_{\text {wave }}$ as the wavelength utilization factor. With $\pi\left(N_{r}, J_{l}\right), P_{\text {light }}$ and $P_{\text {wave }}$ are expressed as

$$
\begin{aligned}
& P_{\text {light }}=\sum_{\left(N_{r}, J_{l}\right) \in U} \sum_{i=1}^{W-1} 1_{\left\{0<J_{l_{i}} \leq K_{l}\right\}} \frac{\pi\left(N_{r}, \boldsymbol{J}_{l}\right)}{W-1}, \\
& P_{\text {wave }}=\sum_{\left(N_{r}, \boldsymbol{J}_{l}\right) \in U}\left\{1_{\left\{N_{r}>0\right\}}+\sum_{i=1}^{W-1} 1_{\left\{0<J_{l_{i}} \leq K_{l}\right\}}\right\} \frac{\pi\left(N_{r}, \boldsymbol{J}_{l}\right)}{W} .
\end{aligned}
$$

\section{Performance Analysis in Heavy Traffic Case}

In this section, we analyze the performance of our method in the heavy traffic case.

\subsection{System model}

Since the establishment/release of lightpaths rarely occurs and each node receives the same volume of traffic, we assume that each node maintains $r$ lightpaths. As a result, we have an $\mathrm{M} / \mathrm{G} / 1 / K_{r}$ queue for the layer 3 routing kernel and $r \mathrm{M} / \mathrm{G} / K_{l} / K_{l}$ queues for established lightpaths, respectively (see Figure 10). Note that $W-r-1$ wavelengths are used for lightpaths established by the other nodes.

In our approximation under heavy traffic, $r$ plays an important role to obtain good estimates of performance measures. We give upper and lower bounds of $r$ by considering the combination of lightpaths between any pairs of nodes in the ring network.

We define the length of lightpath as the number of links between source and destination nodes. $r$ reaches its maximum when the number of lightpaths in the ring network is the largest and this occurs in the following way as shown in Figure 11. First, establish the lightpaths whose length equals two with two wavelengths *. Second, establish the lightpaths whose length equals three with the least number of available wavelengths, and so on. Note that all the nodes try to establish lightpaths equally in the symmetric ring network. It is

\footnotetext{
${ }^{*}$ The lightpath whose length equals one is used for the default path.
} 


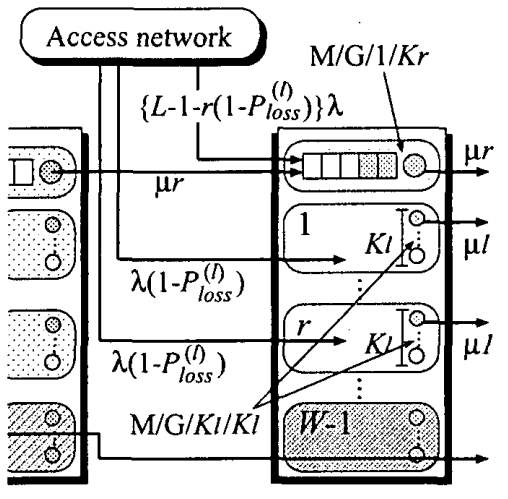

Figure 10: Ring node model with heavy traffic

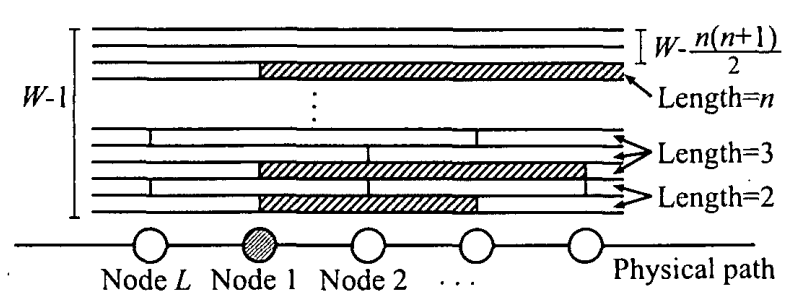

Figure 11: Upper bound of the number of established wavelengths

easy to see that $n$ wavelengths should be used if all the nodes establish the lightpaths with length equal to $n$. Since there are $W-1$ wavelengths, the maximum length $n$ is given by

$$
n=\max \left\{i: \frac{i^{2}+i-2}{2} \leq W-1\right\} .
$$

Each node can establish $n-1$ lightpaths, however, it cannot always establish a lightpath with length equal to $n+1$ due to the shortage of available wavelengths.

Next we estimate the effect of the wavelengths which are not used in the above procedure. In each node, the number of the wavelengths which are not used for lightpaths is

$$
W-1-\frac{n^{2}+n-2}{2}=W-\frac{n(n+1)}{2} .
$$

There are $L$ nodes and hence $L$ links in the ring network. The number of the lightpaths with length equal to $n+1$ in the network is given by

$$
\frac{L}{n+1}\left\{W-\frac{n(n+1)}{2}\right\}
$$

and hence the effect of the above per node is roughly estimated by

$$
\frac{1}{n+1}\left\{W-\frac{n(n+1)}{2}\right\}
$$

Combining the above results yields the upper bound of $r$ as

$$
r \leq n-1+\frac{1}{n+1}\left\{W-\frac{n(n+1)}{2}\right\}
$$

To obtain the lower bound of $r$, we consider a wasteful use of wavelengths. The most wasteful way is the establishment of the lightpaths with length equal to $L-2$. In this case, we have two lightpaths in a wavelength: one is the path with length equal to $L-2$ and the other is that with length equal to two (see Figure 12).

Since the number of lightpaths established in the network is $2(W-1)$, the effect per node is given by $2(W-1) / L$. That is,

$$
r \geq \frac{2(W-1)}{L}
$$




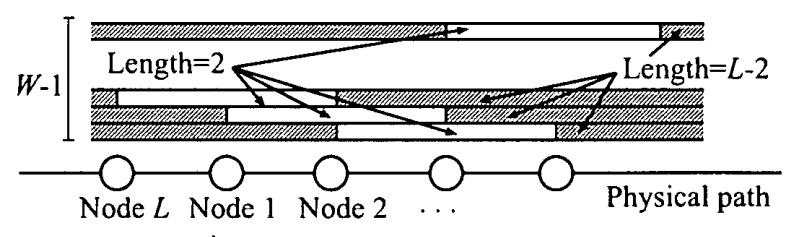

Figure 12: Lower bound of the number of established wavelengths

From (5.2) and (5.3), we finally obtain the range of $r$ as follows:

$$
\frac{2(W-1)}{L} \leq r \leq n-1+\frac{1}{n+1}\left\{W-\frac{n(n+1)}{2}\right\}
$$

As is the case with the light traffic case, we have two kinds of packet flow traffic that arrives at the node: one is from the access network and the other is from the previous node. First we consider packet flow traffic coming from the access network. Since $r$ lightpaths are established, a packet flow from the access network arrives at the routing kernel or one of the established lightpaths. A packet flow arrives at the routing kernel according to a Poisson process with rate $(L-1-r) \lambda$ while it arrives at the established lightpath according to a Poisson process with rate $\lambda$.

The packet flow which arrives at the lightpath tries to establish a new cut-through LSP in the lightpath. If a new cut-through LSP is not established due to the shortage of bandwidth, the packet flow is forwarded to the routing kernel for the transmission with the default path. Let $P_{\text {loss }}^{(l)}$ denote the probability that this cut-through LSP establishment fails at the packet-flow arriving point. With the $\mathrm{M} / \mathrm{G} / K_{l} / K_{l}$ queueing model, $P_{\text {loss }}^{(l)}$ is given by

$$
P_{l o s s}^{(l)}=\frac{\left(\lambda / \mu_{l}\right)^{K_{l}} / K_{l} !}{\sum_{k=0}^{K_{l}}\left(\lambda / \mu_{l}\right)^{k} / k !}
$$

where $1 / \mu_{l}$ is the mean transmission time of a packet flow for a cut-through LSP.

Since the packet flow which fails in establishing a new cut-through LSP in the lightpath is forwarded to the routing kernel with rate $P_{\text {loss }}^{(l)} \lambda$, we assume that packet flows arrive at the routing kernel from its access network according to a Poisson process with rate $\left\{L-1-r\left(1-P_{\text {loss }}^{(l)}\right)\right\} \lambda$.

Next we consider packet flow traffic from the previous node. Because the packet flow is transmitted from the previous node to the tagged node with the default path all the time under heavy traffic, we assume that the packet flow leaves the previous node according to a Poisson process with rate $\mu_{r}$. Thus the whole arrival rate of packet flows at the node, $\lambda_{\text {all }}$, is given by

$$
\lambda_{\text {all }}=(L-1) \lambda+\mu_{r}
$$

Finally, the arrival rate of the packets at the routing kernel in the tagged node, $\lambda_{r}$, is given by

$$
\lambda_{r}=\left\{L-1-r\left(1-P_{\text {loss }}^{(l)}\right)\right\} \lambda+\mu_{r}
$$

\subsection{Performance analysis}

In this subsection, we derive performance measures of the dynamic lightpath configuration method in the case of heavy traffic. As shown in Figure 10, we consider an $\mathrm{M} / \mathrm{G} / 1 / K_{r}$ queue and $r \mathrm{M} / \mathrm{G} / K_{l} / K_{l}$ queues. 
Let $\rho_{r}$ and $\rho_{r}^{\prime}$ denote the offered and carried loads of the routing kernel, respectively. We have

$$
\rho_{r}=\frac{\lambda_{r}}{\mu_{r}}
$$

where $\lambda_{r}$ is given by (5.7). We define $\pi_{0}^{r}$ as the steady state probability that there are no packet flows in the routing kernel. Then $\rho_{r}^{\prime}$ is expressed as [17]

$$
\rho_{r}^{\prime}=\frac{\rho_{r}}{\pi_{0}^{r}+\rho_{r}}
$$

Since a packet flow is lost only at the routing kernel and hence the loss probability $P_{\text {loss }}$ is given by

$$
P_{\text {loss }}=\left\{1-\frac{1}{\pi_{0}^{r}+\rho_{r}}\right\} \frac{\lambda_{r}}{\lambda_{\text {all }}} .
$$

Moreover, the wavelength utilization factor $P_{\text {wave }}$ is expressed as

$$
P_{\text {wave }}=\frac{\rho_{r}^{\prime}+r\left\{1-\frac{1}{\sum_{k=0}^{K_{l}}\left(\lambda / \mu_{l}\right)^{k} / k !}\right\}}{W} .
$$

We can calculate $\pi_{0}^{r}$ in a recursive procedure [17].

Remark. Since $r$ is defined as the number of established lightpaths at the node, $r$ should take integer value. However, $P_{\text {loss }}$ and $P_{\text {wave }}$ are approximations and it is not clear whether non-integer $r$ greatly affects $P_{\text {loss }}$ and $P_{\text {wave }}$, or not. Therefore, for the calculations of $P_{\text {loss }}$ and $P_{\text {wave }}$, we use (5.4) which takes real values.

\section{Numerical Examples}

In this section, we show some numerical results calculated by the approximation analysis and simulation. In the simulation, we assume that the lightpath establishment/release time, $1 / p$, and the extra holding time, $1 / h$, are constant, while they are exponentially distributed with the means $1 / p$ and $1 / h$ in the approximation analysis.

We assume that the bandwidth of a wavelength, $B$, is equal to $10 \mathrm{Gbps}$ and a packet flow contains 10 packets whose sizes are 1,250 bytes on average ${ }^{\dagger}$. Hence the size of a packet flow is exponentially distributed with the mean $\delta=100,000$ bits.

\subsection{Light traffic case}

In this subsection, we show numerical results in the case of light traffic. Here, performance measures are calculated with the analysis results of Section 4.

\subsubsection{Impact of processing speed of the routing kernel}

First we consider how the processing speed of the routing kernel affects the packet-flow loss probability and wavelength utilization factor. We set $W=4, K_{r}=5, T_{h}=3$, and $L=10$. In this network, we assume that each wavelength supports cut-through LSPs with fixed bandwidth $B_{l}=2.5 \mathrm{Gbps}$. Hence the number of cut-through LSPs in a lightpath, $K_{l}$, is equal to 4 and the mean transmission time of a cut-through LSP, $1 / \mu_{l}$, becomes equal to $2.5 \mu \mathrm{s}$. In addition, we assume that both the mean lightpath establishment/release time $1 / p$ and the mean extra holding time $1 / h$ are equal to $10 \mathrm{~ms}$.

\footnotetext{
${ }^{\dagger}$ We investigated the case where the average number of packets in a packet flow is 1000 and obtained the tendency similar to those in the case where the number of packets is 10 . In this case, however, lightpaths are rarely established and released, and hence the impact of the lightpath establishment/release time becomes small.
} 


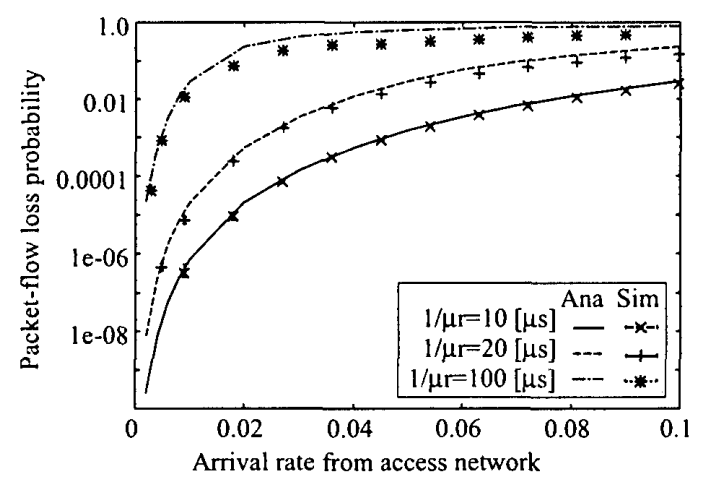

(a) Packet-flow loss probability

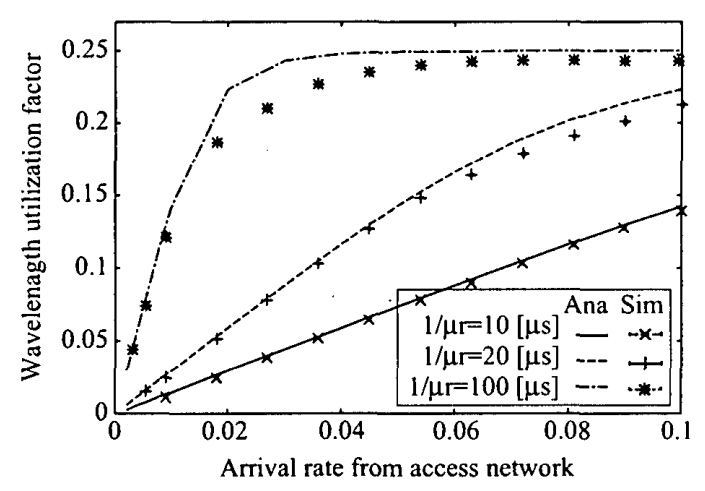

(b) Wavelength utilization factor

Figure 13: Packet-flow loss probability and wavelength utilization factor vs. arrival rate from access network in the light traffic case

Figures 13(a) and (b) show the packet-flow loss probability and wavelength utilization factor, respectively, against the arrival rate of packet flows in the cases of $1 / \mu_{r}=10,20$, and $100 \mu \mathrm{s}$.

From Figure 13(a), we observe that the loss probability calculated with the approximation analysis is almost the same as that with the simulation when the processing speed of the routing kernel is $10 \mu \mathrm{s}$. On the other hand, we can see the discrepancy between the analysis and simulation results when the processing speed of the routing kernel becomes large. This is because a large processing time of the routing kernel causes a large loss probability and our assumption does not hold. However, our approximation analysis is useful for the calculation of the loss probability especially when the loss probability is smaller than 0.05 .

In addition, from Figure 13(b), we observe that the analytical model is useful for the calculation of the wavelength utilization factor when the wavelength utilization factor is smaller than 0.15. Therefore, our approximation analysis in the case of light traffic is effective.

From both figures, we observe that the large processing time of the routing kernel gives a large loss probability and a large wavelength utilization factor. This is because the large processing time of the routing kernel causes congestion and this results in the increase of the number of established lightpaths.

In the following subsections for the light traffic case, only analytical results are shown because the analytical and simulation results are almost the same.

\subsubsection{Impact of the bandwidth of cut-through LSPs}

Next we investigate how the bandwidth of cut-through LSPs in a lightpath affects the packet-flow loss probability and the wavelength utilization factor. We assume that $W=2$, $K_{r}=5, T_{h}=1, L=10,1 / \mu_{r}=10 \mu \mathrm{s}$, and $1 / h=10 \mathrm{~ms}$. In terms of the arrival rate of packet flows from access network, we set $(L-1) \lambda=0.05$.

Figures 14(a) and (b) show the packet-flow loss probability and wavelength utilization factor, respectively, against the bandwidth of cut-through LSPs in the cases of $1 / p=0.1$, 1,10 , and $100 \mathrm{~ms}$. Here, the bandwidths of cut-through LSPs, $B_{l}$, are set to 50, 153.8, 625, 1250 , and $2500 \mathrm{Mbps}$, i.e., the numbers of cut-through LSPs in a lightpath, $K_{l}$, are 200, 65, 16,8 , and 4 .

From these figures, we observe that smaller bandwidth of a cut-through LSP gives a 


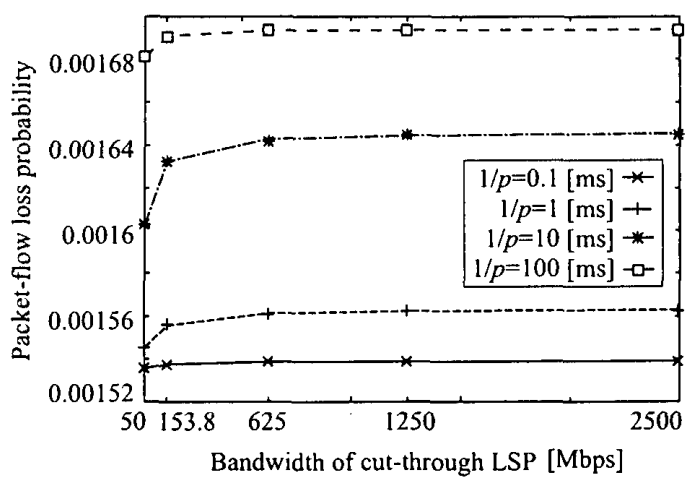

(a) Packet-flow loss probability

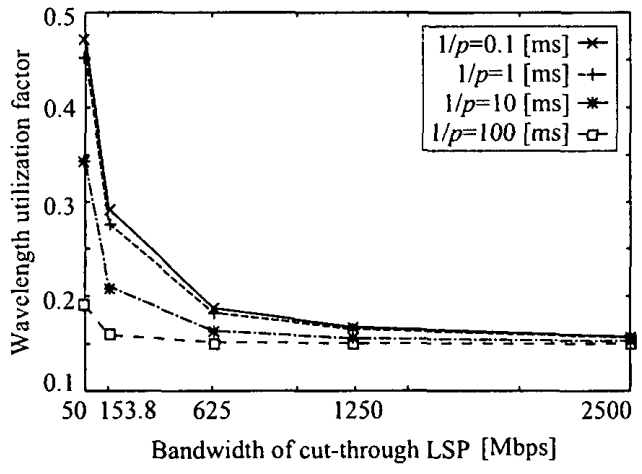

(b) Wavelength utilization factor

Figure 14: Packet-flow loss probability and wavelength utilization factor vs. bandwidth of cut-through LSP in the light traffic case

smaller loss probability and a larger wavelength utilization factor. In particular, from Figure 14(b), we observe that the small bandwidth of LSPs in a lightpath gives a large wavelength utilization factor as the lightpath establishment/release time becomes small. This implies that multiple LSPs with small bandwidth effectively utilize the large bandwidth of a lightpath.

\subsubsection{Impact of congestion threshold}

Next, we investigate how the congestion threshold affects the packet-flow loss probability and lightpath utilization factor. We assume that $W=4, K_{r}=10, L=10,1 / \mu_{r}=10$, and $1 / h=10 \mathrm{~ms}$. In addition, we assume that $B_{l}=10 \mathrm{Gbps}$ and $(L-1) \lambda=0.1$.

Figures 15(a) and (b) show the packet-flow loss probability and lightpath utilization factor, respectively, against the threshold in the cases of $1 / p$ set to $1,10,100$, and $1000 \mathrm{~ms}$. In addition, we indicate the optimal thresholds which achieve the smallest loss probability and the largest lightpath utilization factor in both figures.

From Figure 15(a), we find that the values of $T_{h}$ equal to $1,1,3$, and 5 give the smallest loss probability in the cases of $1 / p=1,10,100$ and $1000 \mathrm{~ms}$, respectively. Moreover, in Figure 15(b), the same thresholds also give the largest lightpath utilization factor. That is, the optimal thresholds in terms of both performance measures are the same.

When the threshold is smaller than the optimal threshold, congestion occurs frequently and this results in frequent lightpath establishment and release. Note that both the loss probability and the lightpath utilization factor do not degrade so much even though the wavelength can not be used during the lightpath establishment/release time.

On the other hand, as the threshold becomes larger than the optimal threshold, the congestion rarely occurs. If the congestion does not occur, most of arriving packet flows are transmitted with the default path. This causes a large loss probability and a small lightpath utilization factor.

Moreover, as the lightpath establishment/release time becomes small, the impact of the threshold on both performance measures becomes large. Therefore, it is important to design the threshold carefully in order to achieve a small loss probability and a large lightpath utilization factor.

\subsubsection{Impact of extra holding time}

In this subsection, we consider how the extra holding time affect the packet-flow loss probability and wavelength utilization factor. We assume that $W=4, K_{r}=5, T_{h}=1, L=10$, 


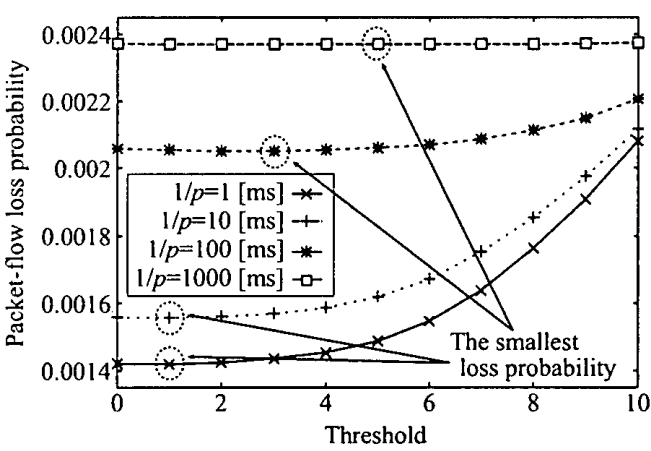

(a) Packet-flow loss probability

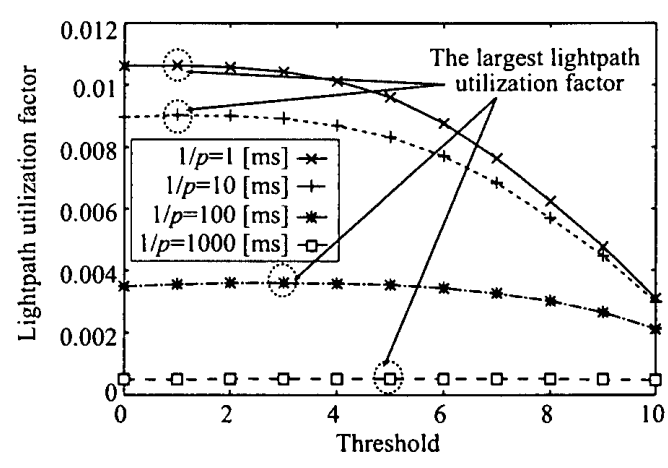

(b) Lightpath utilization factor

Figure 15: Packet-flow loss probability and lightpath utilization factor vs. threshold in the light traffic case

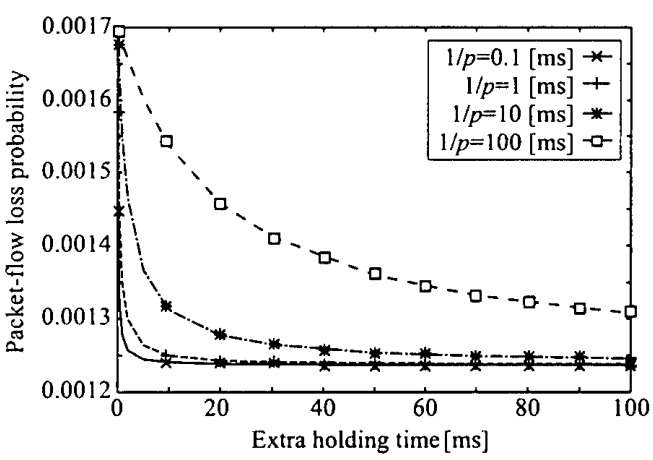

(a) Packet-flow loss probability

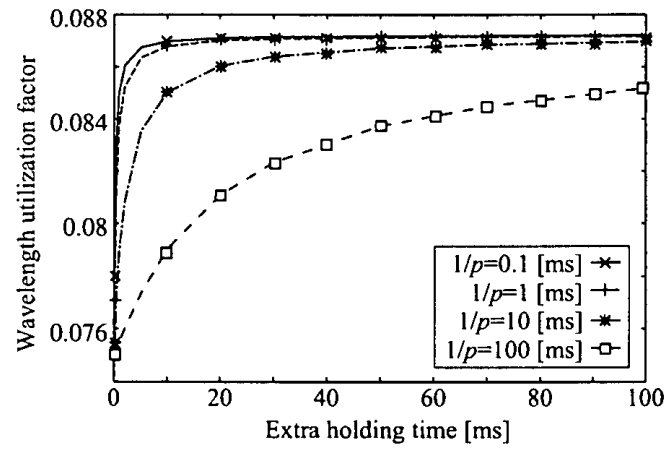

(b) Wavelength utilization factor

Figure 16: Packet-flow loss probability and wavelength utilization factor vs. extra holding time in the light traffic case

and $1 / \mu_{r}=10 \mu \mathrm{s}$. We also assume that $B_{l}=2.5 \mathrm{Gbps}$ and $(L-1) \lambda=0.05$.

Figures 16(a) and (b) show the loss probability and wavelength utilization factor, respectively, against the extra holding time in the cases of $1 / p=0.1,1,10$, and $100 \mathrm{~ms}$.

From Figure 16(a), we observe that the packet-flow loss probability decreases as the extra holding time becomes large. In addition, the loss probability becomes large when the lightpath establishment/release time is large. This is because a wavelength can not be used during the lightpath establishment/release time and frequent establishment and release of lightpaths waste the time.

However, a small extra holding time becomes efficient for the loss probability when the lightpath establishment/release time is in the order of $10 \mathrm{~ms}$. If the lightpath establishment/release time is $10 \mathrm{~ms}$, the extra holding time of $20 \mathrm{~ms}$ is enough for providing a small loss probability.

From Figure 16(b), we observe that the wavelength utilization factor increases as the extra holding time becomes large. In addition, we find that the wavelength utilization factor is small when the lightpath establishment/release time is large. A small extra holding time becomes efficient for the wavelength utilization factor when the lightpath establishment/release time is in the order of $10 \mathrm{~ms}$. If the lightpath establishment/release time is $10 \mathrm{~ms}$, the extra holding time of $20 \mathrm{~ms}$ is also enough for providing a large wavelength utilization factor. 


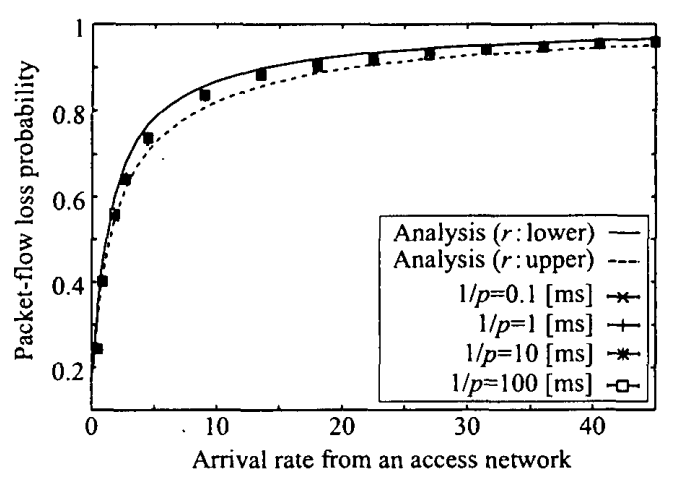

(a) Packet-flow loss probability

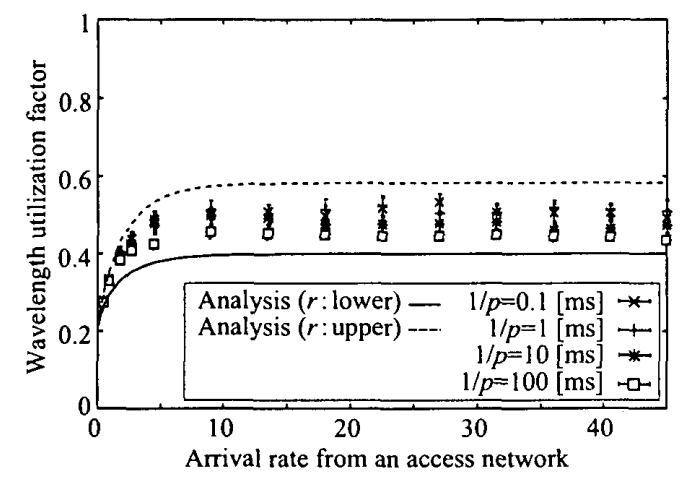

(b) Wavelength utilization factor

Figure 17: Packet-flow loss probability and wavelength utilization factor vs. arrival rate in the heavy traffic case, $1 / h=10 \mathrm{~ms}$

From the above observations, a small extra holding time can provide a small loss probability and a large wavelength utilization factor when the lightpath establishment/release time is in the order of $10 \mathrm{~ms}$. The extra holding time should be set to $20 \mathrm{~ms}$ rather than $100 \mathrm{~ms}$ in the case of $1 / p=10 \mathrm{~ms}$, because the small extra holding time is effective for an asymmetric ring network.

\subsection{Heavy traffic case}

In this subsection, we present numerical examples of the packet-flow loss probability and wavelength utilization factor for the heavy traffic case. We assume that $W=4, K_{r}=5$, $T_{h}=3, L=10$, and $1 / \mu_{r}=10 \mu \mathrm{s}$. We also set $B_{l}=2.5 \mathrm{Gbps}$.

\subsubsection{Impact of lightpath establishment/release time}

We investigate how the lightpath establishment/release time affects the packet-flow loss probability and wavelength utilization factor.

Figures 17(a) and (b) illustrate the packet-flow loss probability and wavelength utilization factor, respectively, against the arrival rate from the access network with $1 / h=10 \mathrm{~ms}$ and $1 / p=0.1,1,10$, and $100 \mathrm{~ms}$. From Figures $17(\mathrm{a})$ and (b), we observe that the results of simulation lie between the curves of the upper and lower bounds. Note that in Figure 17(a), the upper bound value of $r$ gives the lower bound of the packet-flow loss probability, while the lower bound of $r$ gives the upper bound of the packet-flow loss probability. On the other hand, in Figure 17(b), the upper bound value of $r$ gives the upper bound of the wavelength utilization factors and vice versa.

In Figure 17(a), the simulation results close to the upper bound regardless of the establishment/release time. Note that the upper bound of the packet-flow loss probability is calculated with the lower bound of $r$ equal to $2(W-1) / L$. That is, the lower bound of $r$ succeeds in the prediction of loss behavior at packet-flow level under heavy traffic.

On the other hand, we observe in Figure 17(b) that the simulation results become close to the lower bound when the establishment/release time becomes large. However, the discrepancy between the upper and lower bounds is relatively large.

Figures 17(a) and (b) show that the packet-flow loss probability and the wavelength utilization factor do not change so much when the lightpath establishment/release time becomes large. This is because the established lightpaths are not released frequently due to the heavy traffic. 


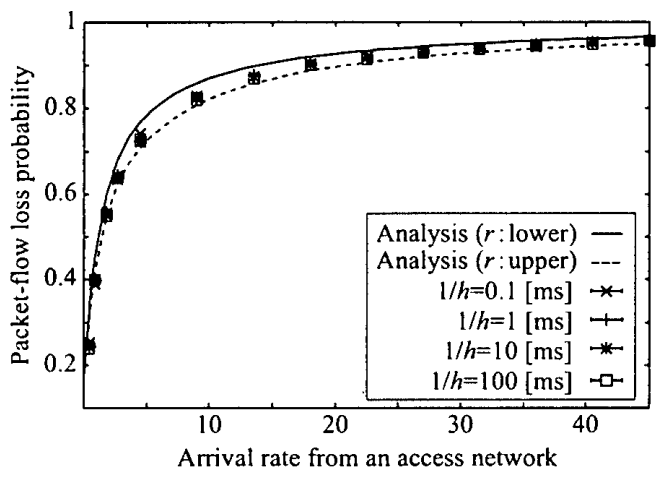

(a) Packet-flow loss probability

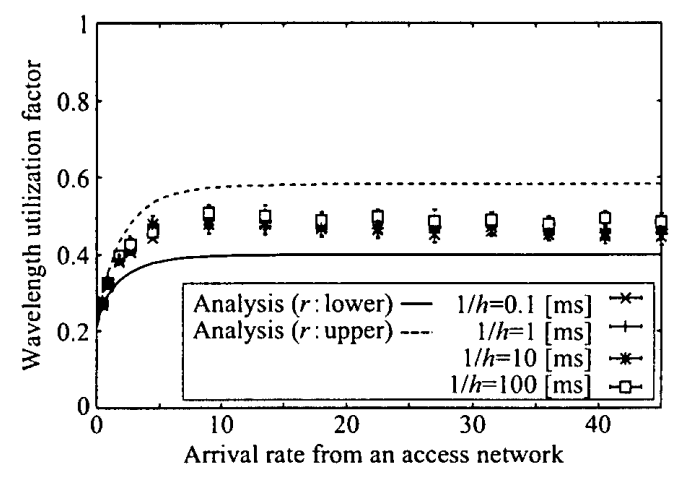

(b) Wavelength utilization factor

Figure 18: Packet-flow loss probability and wavelength utilization factor vs. arrival rate in the heavy traffic case, $1 / p=10 \mathrm{~ms}$

\subsubsection{Impact of extra holding time}

Finally, we investigate how the extra holding time affects the packet-flow loss probability and the wavelength utilization factor. Figures $18(\mathrm{a})$ and (b) show the packet-flow loss probability and the wavelength utilization factor, respectively, against the arrival rate with $1 / p=10 \mathrm{~ms}$ and $1 / h=0.1,1,10$, and $100 \mathrm{~ms}$.

From Figure 18(a), simulation results lie between upper and lower bounds. As is the case with Figure 17(a), the upper bound gives the good estimate of the packet-flow loss probability. From Figure 18(b), we also observe the same tendency as Figure 17(b). As a result, the upper and lower bounds for the packet-flow loss probability gives good estimates, while those for the wavelength utilization factor fails in providing accurate estimates. Further improvement is needed for the accurate estimation of the wavelength utilization factor.

\section{Conclusion}

In this paper, we considered the dynamic lightpath configuration method where a lightpath supports multiple LSPs, and analyzed the loss probability of packet flows and the wavelength utilization factor under light and heavy traffic conditions for symmetric WDM ring networks.

Numerical results in the light traffic case showed that our approximation analysis gives good estimates for the packet-flow loss probability and wavelength utilization factor. With multiple LSPs in a lightpath, the dynamic lightpath configuration method becomes effective when the lightpath establishment/release time becomes small. As for the design of the threshold in the method, the optimal thresholds which give the smallest loss probability and the largest lightpath utilization factor can be obtained from the light traffic analysis. It is important to design the threshold carefully in order to achieve a small loss probability and a large lightpath utilization factor. We also observed in the light traffic case that a small extra holding time is effective when the lightpath establishment/release time is in the order of $10 \mathrm{~ms}$.

In the heavy traffic case, we showed that our approximation analysis with the lower bound of $r$ is useful to estimate the packet-flow loss probability. On the other hand, the resulting estimates of the wavelength utilization factor are not accurate. Further improvement of the approximation is needed for the well estimation of the wavelength utilization factor. 


\section{Acknowledgement}

This research was supported in part by the International Communications Foundation (ICF), and by Ministry of Education, Culture, Sports, Science and Technology under Grantin-Aid for Young Scientists No. 15760264.

\section{References}

[1] D. O. Awduche and Y. Rekhter: Multiprotocol lambda switching: combining MPLS traffic engineering control with optical crossconnects. IEEE Communications Magazine, 39 (2001), 111-116.

[2] P. Bonenfant and A. R. Moral: Optical data networking. IEEE Communications Magazine, 38 (2000), 63-70.

[3] T. Chikama, H. Onaka, and S. Kuroyanagi: Photonic networking using optical add drop multiplexers and optical cross-connects. FUJITSU Scientific \& Technical Journal, 35 (1999), 46-55.

[4] I. Chlamtac, V. Elek, A. Fumagalli, and C. Szabó: Scalable WDM access network architecture based on photonic slot routing. IEEE/ACM Transactions on Networking, 7 (1999), 1-9.

[5] O. Gerstel, R. Ramaswami, and G. H. Sasaki: Cost-effective traffic grooming in WDM rings. IEEE/ACM Transactions on Networking, 8 (2000), 618-630.

[6] O. Gerstel, G. H. Sasaki, S. Kutten, and R. Ramaswami: Worst-case analysis of dynamic wavelength allocation in optical networks. IEEE/ACM Transactions on Networking, 7 (1999), 833-846.

[7] N. Ghani, S. Dixit, and T. S. Wang: On IP-over-WDM integration. IEEE Communications Magazine, 38 (2000), 72-84.

[8] L. Kleinrock: Queueing Systems, Volume I: Theory (John Willey \& Sons, New York, $1975)$.

[9] M. W. McKinnon, H. G. Perros, and G. N. Rouskas: Performance analysis of broadcast WDM networks under IP traffic. Performance Evaluation, 36-37 (1999), 333-358.

[10] Y. Miyao: $\lambda$-ring system: an application in survivable WDM networks of interconnected self-healing ring systems. IEICE Transactions on Communications, E84-B (2001), 1596-1604.

[11] S. Nakazawa, H. Tamura, K. Kawahara, and Y. Oie: Performance analysis of IP datagram transmission delay in MPLS: impact of both number and bandwidth of LSPs of layer 2. IEICE Transactions on Communications, E85-B (2002), 165-172.

[12] B. Ramamurthy and B. Mukherjee: Wavelength conversion in WDM networking. IEEE Journal on Selected Areas in Communications, 16 (1998), 1061-1073.

[13] R. Ramaswami and K. N. Sivarajan: Optical Networks: A Practical Perspective (Morgan Kaufmann Publishers, San Francisco, 1998).

[14] K. Sato, S. Okamoto, and H. Hadama: Network performance and integrity enhancement with optical path layer technologies. IEEE Journal on Selected Areas in Communications, 12 (1994), 159-170.

[15] K. Struyve, N. Wauters, P. Falcao, P. Arijs, D. Colle, P. Demeester, and P. Lagasse: Application, design, and evolution of WDM in GTS's pan-european transport network. IEEE Communications Magazine, 38 (2000), 114-121. 
[16] T. Tachibana and S. Kasahara: Performance analysis of dynamic lightpath configuration for WDM asymmetric ring networks, In Proceedings of IFIP Networking 2002 (2002), 972-983.

[17] H. Takagi: Queueing Analysis: A Foundation of Performance Evaluation, Volume 2: Finite Systems (Elsevier Science Publishers B, Amsterdam, 1993).

[18] W. Weiershausen, A. Mattheus, and F. Küppers: Realisation of next generation dynamic WDM networks by advanced OADM design. D. W. Faulkner and A. L. Harmer (eds.): WDM and Photonic Networks (IOS Press, Amsterdam, 2000), 199-207.

[19] R. W. Wolff: Stochastic Modeling and the Theory of Queues (Pretice Hall, New Jersey, 1989).

[20] Y. Yuki, M. Nakao, and H. Ibe: Study of wavelength path configuration method for WDM networks. In Proceedings of the 2000 IEICE Society Conference (2000), B-10-123 (in Japanese).

\section{A. Equilibrium State Equations}

For simplicity, we consider the case of $W=2$. Then $\pi\left(N_{r}, J_{l_{1}}\right)$ 's satisfy the following equilibrium state equations.

(A) $J_{l_{1}}=I: l_{1}$ is idle.

$$
\begin{array}{rlrl}
\lambda_{\text {all }} \pi(0, I)= & \mu_{r} \pi(1, I)+p \pi(0, R), & \\
\left(\lambda_{\text {all }}+\mu_{r}\right) \pi\left(N_{r}, I\right)= & \lambda_{\text {all }} \pi\left(N_{r}-1, I\right)+\mu_{r} \pi\left(N_{r}+1, I\right) \\
& +p \pi\left(N_{r}, R\right), \quad\left(0<N_{r} \leq T_{h}\right), \\
\left(\lambda_{\text {all }}+\mu_{r}\right) \pi\left(N_{r}, I\right)= & \mu_{r} \pi\left(N_{r}+1, I\right) \\
& +p \pi\left(N_{r}, R\right), & \\
\left(\lambda_{\text {all }}+\mu_{r}\right) \pi\left(K_{r}, I\right)= & p \pi\left(K_{r}, R\right) .
\end{array}
$$

(B) $J_{l_{1}}=S: l_{1}$ is being established.

$$
\begin{aligned}
\left(\lambda_{\text {all }}+p\right) \pi(0, S)= & \mu_{r} \pi(1, S), \\
\left(\lambda_{\text {all }}+\mu_{r}+p\right) \pi\left(N_{r}, S\right)= & \lambda_{\text {all }} \pi\left(N_{r}-1, S\right) \\
& +\mu_{r} \pi\left(N_{r}+1, S\right), \quad\left(0<N_{r} \leq T_{h}\right), \\
\left(\lambda_{\text {all }}+\mu_{r}+p\right) \pi\left(N_{r}, S\right)= & \lambda_{\text {all }} \pi\left(N_{r}-1, S\right)+\lambda_{\text {all }} \pi\left(N_{r}-1, I\right) \\
& +\mu_{r} \pi\left(N_{r}+1, S\right), \quad\left(T_{h}<N_{r}<K_{r}\right), \\
\left(\mu_{r}+p\right) \pi\left(K_{r}, S\right)= & \lambda_{\text {all }} \pi\left(K_{r}-1, S\right)+\lambda_{\text {all }} \pi\left(K_{r}-1, I\right) \\
& +\lambda_{\text {all }} \pi\left(K_{r}, I\right) .
\end{aligned}
$$

(C) $J_{l_{1}}=n\left(0 \leq n \leq K_{l}\right): l_{1}$ is busy.

(a) $n=0$

$$
\begin{aligned}
\left(\dot{\lambda}_{\text {all }}+h\right) \pi(0,0)= & \mu_{r} \pi(1,0)+\mu_{l} \pi(0,1)+p \pi(0, S) \\
\left(\lambda_{\text {all }}+\mu_{r}+h\right) \pi\left(N_{r}, 0\right)= & \left(\lambda_{\text {all }}-\lambda\right) \pi\left(N_{r}-1,0\right) \\
& +\mu_{r} \pi\left(N_{r}+1,0\right)+\mu_{l} \pi\left(N_{r}, 1\right) \\
& +p \pi\left(N_{r}, S\right), \quad\left(0<N_{r}<K_{r}\right), \\
\left(\lambda+\mu_{r}+h\right) \pi\left(K_{r}, 0\right)= & \left(\lambda_{\text {all }}-\lambda\right) \pi\left(K_{r}-1,0\right)+\mu_{l} \pi\left(K_{r}, 1\right) \\
& +p \pi\left(K_{r}, S\right) .
\end{aligned}
$$


(b) $0<n<K_{l}$

$$
\begin{aligned}
\left(\lambda_{\text {all }}+\mu_{l}\right) \pi(0, n)= & \mu_{r} \pi(1, n)+\lambda \pi(0, n-1) \\
& +\mu_{l} \pi(0, n+1) \\
\left(\lambda_{\text {all }}+\mu_{r}+\mu_{l}\right) \pi\left(N_{r}, n\right)= & \left(\lambda_{\text {all }}-\lambda\right) \pi\left(N_{r}-1, n\right) \\
& +\mu_{r} \pi\left(N_{r}+1, n\right)+\lambda \pi\left(N_{r}, n-1\right) \\
& +\mu_{l} \pi\left(N_{r}, n+1\right), \quad\left(0<N_{r}<K_{r}\right), \\
\left(\lambda+\mu_{r}+\mu_{l}\right) \pi\left(K_{r}, n\right)= & \left(\lambda_{\text {all }}-\lambda\right) \pi\left(K_{r}-1, n\right) \\
& +\lambda \pi\left(K_{r}, n-1\right)+\mu_{l} \pi\left(K_{r}, n+1\right) .
\end{aligned}
$$

(c) $n=K_{l}$

$$
\begin{aligned}
\left(\lambda_{\text {all }}+\mu_{l}\right) \pi\left(0, K_{l}\right)= & \mu_{r} \pi\left(1, K_{l}\right)+\lambda \pi\left(0, K_{l}-1\right), \\
\left(\lambda_{\text {all }}+\mu_{r}+\mu_{l}\right) \pi\left(N_{r}, K_{l}\right)= & \lambda_{\text {all }} \pi\left(N_{r}-1, K_{l}\right)+\mu_{r} \pi\left(N_{r}+1, K_{l}\right) \\
& +\lambda \pi\left(N_{r}, K_{l}-1\right), \quad\left(0<N_{r}<K_{r}\right), \\
\left(\mu_{r}+\mu_{l}\right) \pi\left(K_{r}, K_{l}\right)= & \lambda_{\text {all }} \pi\left(K_{r}-1, K_{l}\right)+\lambda \pi\left(K_{r}, K_{l}-1\right) .
\end{aligned}
$$

(D) $J_{l_{1}}=R: l_{1}$ is being released.

$$
\begin{aligned}
\left(\lambda_{\text {all }}+p\right) \pi(0, R)= & \mu_{r} \pi(1, R)+h \pi(0,0) \\
\left(\lambda_{\text {all }}+\mu_{r}+p\right) \pi\left(N_{r}, R\right)= & \lambda_{\text {all }} \pi\left(N_{r}-1, R\right)+\mu_{r} \pi\left(N_{r}+1, R\right) \\
& +h \pi\left(N_{r}, 0\right), \quad\left(0<N_{r}<K_{r}\right), \\
\left(\mu_{r}+p\right) \pi\left(K_{r}, R\right)= & \lambda_{\text {all }} \pi\left(K_{r}-1, R\right)+h \pi\left(K_{r}, 0\right) .
\end{aligned}
$$

\section{Shoji Kasahara}

Graduate School of Information Science Nara Institute of Science and Technology Takayama 8916-5, Ikoma Nara 630-0192, Japan .

Email: kasahara@is.naist.jp 